\title{
Scintigraphic validation of AC Biosusceptometry to study the gastric motor activity and the intragastric distribution of food in humans
}

\author{
M. F. AMÉRICO, ${ }^{\star}$ R. B. OLIVEIRA,†F. G. ROMEIRO,† O. BAFFA,§ L. A. CORÁ \& J. R. A. MIRANDA \\ ^Departamento de Fisiologia, FMRP, Universidade de São Paulo - USP, Ribeirão Preto, São Paulo, Brazil \\ $\dagger$ Departamento de Clínica Médica, FMRP, Universidade de São Paulo - USP, Ribeirão Preto, São Paulo, Brazil \\ \$Departamento de Clínica Médica, FMB, Universidade Estadual Paulista - UNESP, Botucatu, São Paulo, Brazil \\ §Departamento de Física e Matemática, FFCLRP, Universidade de São Paulo - USP, Ribeirão Preto, São Paulo, Brazil \\ -Departamento de Física e Biofísica, IBB, Universidade Estadual Paulista - UNESP, Botucatu, São Paulo, Brazil
}

\begin{abstract}
Abnormal intragastric distribution of food (IDF) and a phasic contractility in the proximal stomach have been related to dyspeptic symptoms. Thus, the behaviour of the stomach and the proximal region, in particular, continues to attract attention and demand for reliable and comfortable techniques. The aims of this study were to employ AC Biosusceptometry (ACB) and scintigraphy to evaluate IDF and gastric motor activity in humans. Fifteen healthy volunteers ingested $60 \mathrm{~mL}$ of yogurt containing $2 \mathrm{mCi}$ of ${ }^{99 \mathrm{~m}} \mathrm{Tc}$ and $4 \mathrm{~g}$ of ferrite. Each volunteer had gastric motility and IDF evaluated twice on separate days; on one occasion by $A C B$ and another by scintigraphy. Digital signal processing was performed in MatLab (Mathworks Inc., Natick, MA, USA). Results were expressed as mean $\pm S D$. Similar results of distal accumulation time $(P<0.001)$ were obtained for scintigraphy (6.93 $\pm 3.25 \mathrm{~min})$ and for $A C B \quad(7.04 \pm$ $3.65 \mathrm{~min})$. Fast Fourier Transform revealed two dominant frequencies $(P>0.9)$. Besides the well-know frequency of $3 \mathrm{cpm}$, our results showed identical frequencies in proximal stomach recordings $(P<0.001)$ for scintigraphic $(1.01 \pm 0.01 \mathrm{cpm})$ and $A C B$ $(0.98 \pm 0.06 \mathrm{cpm})$. In summary, our data showed that scintigraphy and $A C B$ are promising techniques to evaluate several aspects of gastric motility. Moreover,
\end{abstract}

Address for correspondence

José Ricardo A. Miranda, Departamento de Física e Biofísica, Instituto de Biociências - IBB, Laboratório de Biomagnetismo, Universidade Estadual Paulista - UNESP, CXP 510, CEP

18618-000, Botucatu, São Paulo, Brazil.

Tel: +55 143811 6254; fax: +55 143811 6346;

e-mail: jmiranda@ibb.unesp.br

Received: 16 February 2007

Accepted for publication: 29 April 2007
$A C B$ is non-invasive, radiation-free and deserves the same importance as conventional methods for this kind of analysis.

Keywords gastric motility, phasic contractions, proximal stomach, volume waves.

\section{INTRODUCTION}

The stomach is composed of two distinct functional regions. ${ }^{1}$ The distal stomach is capable of generating vigorous phasic contractions, which cause reduction in size of ingested particles and subsequent emptying. ${ }^{2}$ The proximal stomach is primarily concerned with storage of the ingested food. During a meal, the stomach continuously adapts its size to the content by gradually relaxing its musculature, performing the so-called accommodation to distension. ${ }^{3}$ However, some aspects of food-induced gastric relaxation, such as the relationship of the magnitude of the proximal stomach response to different volumes are poorly understood, ${ }^{4}$ and it is conceivable that some volume is accommodated in the stomach before the distension reflex is elicited.

Several techniques are currently used to study proximal stomach functions; ${ }^{4}$ none of them meets all the criteria for clinical routine. Intragastric barostat is gold standard technique to evaluate gastric accommodation, ${ }^{5,6}$ but the balloon cause unacceptable discomfort to the patient and may disturb normal physiology. ${ }^{7,8}$ Magnetic resonance imaging (MRI) appears to be as accurate as the barostat technique in determining changes in gastric volume, ${ }^{5}$ but depends on expensive and busy equipment. Single photon emission computed tomography (SPECT) enables 
accurate measurement of the total gastric volume, ${ }^{7}$ but uses radioactive isotopes, and depends on expensive, not widely available equipment and sophisticated softwares.

The partitioning of ingested meals between proximal stomach and distal stomach (intragastric distribution of food, IDF) is related to the gastric accommodation. Abnormal IDF has been reported in a number of clinical conditions, including functional dyspepsia..,10 This abnormality may be a consequence of disturbed proximal stomach accommodation ${ }^{11-13}$ and may play a role in symptom production. ${ }^{14}$ Intragastric distribution of food is traditionally assessed by scintigraphy whose major disadvantage is radiation exposure, especially in prolonged studies. ${ }^{6,15}$ Alternating current Biosusceptometry $(\mathrm{ACB})$ is a non-expensive biomagnetic technique, easy to perform, non-invasive and harmless ${ }^{16}$ and has been employed to study several gastrointestinal (GI) functions, by recording gut content movements. ${ }^{16-21}$

Apart from its well-known tonic motor activity, the proximal stomach exhibits a phasic activity, ${ }^{22}$ consistently demonstrated by means of techniques which detect volume changes, such as barostat ${ }^{3}$ and MRI. ${ }^{5}$ An alteration in these 'volume waves', ${ }^{3}$ whose frequency is around of $0.016 \mathrm{~Hz}$ contractions (one cycle per minute), was recently described in functional dyspepsia. ${ }^{23}$ Since ACB has been successfully employed for GI motor activity recordings in canine stomach ${ }^{21}$ and in human colon, ${ }^{24}$ and it is possible that it may be useful for evaluate the proximal gastric motor activity.

The aims of this study were to determine the accuracy of ACB to evaluate IDF and gastric motor activity in humans, taking scintigraphy as gold standard.

\section{METHODS}

\section{ACB sensor}

The ACB sensor provides non-invasive and real-time data acquisition for GI motility studies. This sensor is able to record the magnetic flux variation obtained from the response of a magnetic tracer ingested when an alternating magnetic field is applied.

The multi-sensor ACB system employed in this study, is an assembly of one pair of excitation coils $(\varphi=11 \mathrm{~cm})$ and seven pairs of detection coils $(\varphi=3.5 \mathrm{~cm})$, coaxially arranged in first order gradiometer. The system works as a double magnetic flux transformer, where the pair (excitation/detection) located more distant from magnetic material acts as reference. The approximation of a magnetic material causes an unbalancing in the magnetic flux, and the signal can be monitored. As the signal depends on the distance between sensor and magnetic material in the gastric cavity, the movements of the gastric wall generated by muscle contractions promote modulations in the signal recorded by the sensors. A more detailed description of ACB device was recently published. ${ }^{16}$

\section{Subjects}

Fifteen healthy volunteers (nine female and six male) with a range in body mass index of $20.3-21.4 \mathrm{~kg} \mathrm{~m}^{-2}$ and an age of 20-40 years participated in the studies. None had history of digestive disease or abdominal surgery. Informed written consent was obtained from each participant. The studies were performed in agreement with Declaration of Helsinki and the local Ethics Committee approved the protocol.

\section{Study protocol}

Each volunteer had gastric motility and IDF evaluated twice on separate days; on one occasion by ACB technique and another by external abdominal scintigraphy. The studies were performed in the morning, after an overnight fast, in a randomized order and were separated by an interval of 1 week.

The test meal consisted of $60 \mathrm{~mL}$ of yogurt $\left(1 \mathrm{kcal} \mathrm{mL}{ }^{-1}\right)$ mixed with $4 \mathrm{~g}$ of ferrite $\left(\mathrm{MnFe}_{2} \mathrm{O}_{4}\right.$, $80 \mu \mathrm{m}<\varphi<125 \mu \mathrm{m})$, an inert magnetic material that is not absorbed by GI tract and devoid of biological side effects. ${ }^{17,25}$

In the scintigraphic studies, the test meal was labelled with $20 \mathrm{MBq}$ of technetium $\left({ }^{99 \mathrm{~m}} \mathrm{Tc}\right)$ coupled with phytate (Phytosid; Sydma Medical Equipament and Reagents, Ribeirão Preto, São Paulo, Brazil) as a non-absorbable carrier. External radioactive markers were taped over xiphoid process and umbilicus, serving as anatomical landmarks for data analysis.

\section{In vitro}

In vitro tests were performed to determinate the homogeneous distribution and stability of ${ }^{99 \mathrm{~m}} \mathrm{Tc}$ and of ferrite in the meal test. ${ }^{26}$ The uniformity of distribution of ${ }^{99 \mathrm{~m}} \mathrm{Tc}$ and of ferrite in the meal test was established by signal of cylindrical phantom containing meal with gamma camera and ACB system acquiring data at regular interval during at least $1 \mathrm{~h}$. The same in vitro test was repeated as above but after adding into the meal $100 \mathrm{~mL}$ of $\mathrm{HCl}$ acid so as to simulate conditions of gastric environment. Homogeneous distribution and stability were confirmed. 


\section{Acquisition and data analysis}

The test meal was consumed within 1 min with the volunteers standing in front of either an ACB system (Fig. 1) or a gamma camera (Sopha Vision, Model DST, Sophycammera; Medical Sopha Vision America, Twinsburg, OH, USA). For ACB, magnetic signals were acquired for $16 \mathrm{~min}$ with sampling rate of $10 \mathrm{~Hz}$ channel ${ }^{-1}$ employing lock-in amplifiers (Stanford Research System, Sunnyvale, CA, USA) and digitalized to an A/D board of 16 bits (PCI-MIO16XE-10; National Instruments Inc., Austin, TX, USA). In scintigraphy, anterior dynamic $(64 \times 64$ pixels) frames of $1 \mathrm{~s}$ were acquired for $16 \mathrm{~min}$ and stored

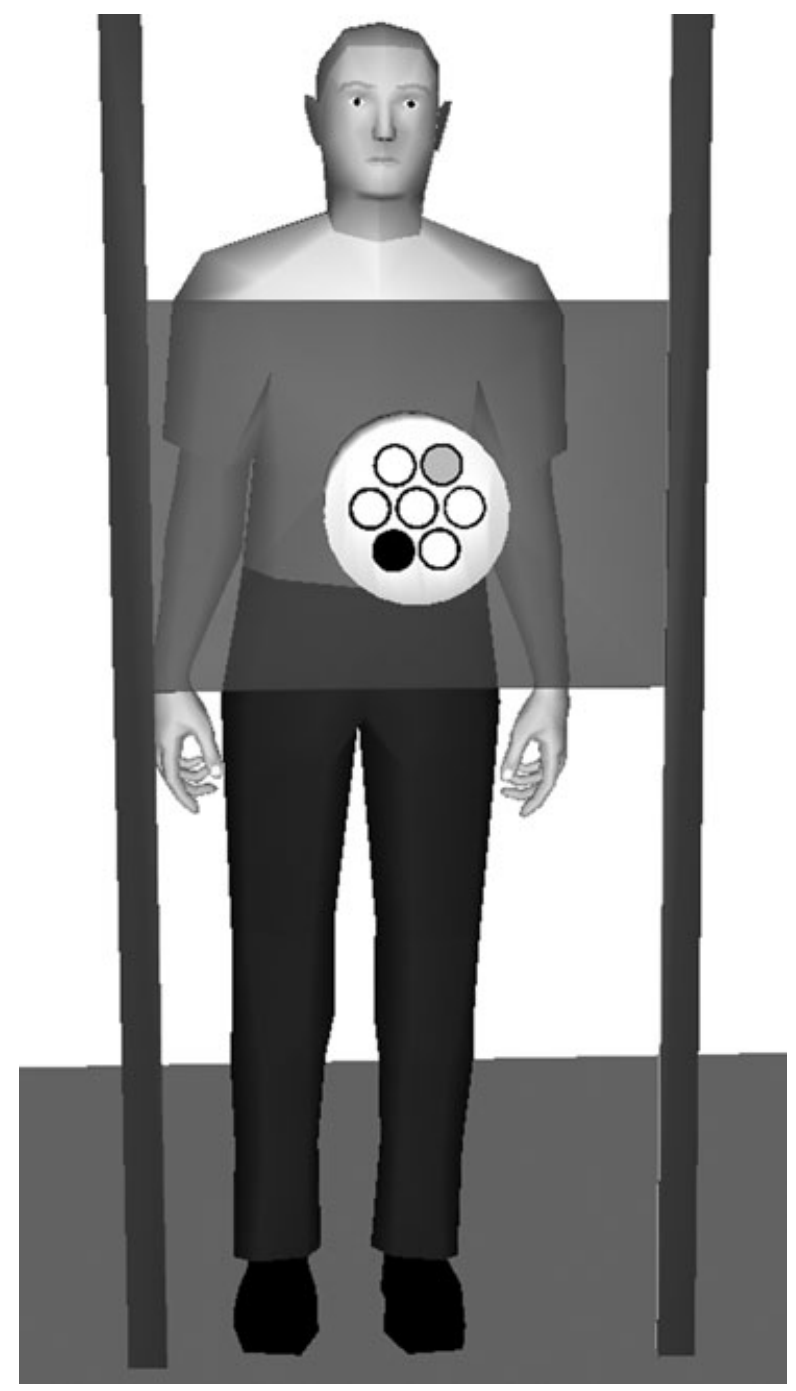

Figure 1 Schematic representation of a volunteer positioned in the multi-sensor Alternating current Biosusceptometry system. Based on this representation, was painted only the sensor and the regions of interest in proximal region (light grey) and distal stomach (black). at a sampling rate of $1 \mathrm{~Hz}$. The gamma camera was set up to record activity around the $140-\mathrm{keV}$ photopeak of technetium-99.

All data were stored in ASCII and analysed in MatLab (Mathworks Inc.). Fast Fourier transform (FFT) was employed to analyse phasic activities in both gastric regions using bi-directional Butterworth low-pass filters with a cutoff frequency at $0.1 \mathrm{~Hz}$.

Seven regions of interest (ROIs) with size and arrangement identical to $\mathrm{ACB}$ multisensor system (Fig. 1) were drawn on the gastric scintigraphic images. Regions of interest were positioned based on anatomical landmarks and this procedure was adopted to provide comparative analyses between both techniques. Plots of 'intensity vs time' and 'counts vs time' were generated for ACB and scintigraphy respectively.

Sigmoidal curves based on Boltzmann function were fitted over tracings corresponding to the distal stomach. Distal accumulation time (DAT) was defined as the time elapsing from the test meal ingestion until the activity (or intensity) reached $99 \%$ of the maximum value in the sigmoidal curves.

\section{Statistical analysis}

Scintigraphic and magnetic data were expressed as mean \pm standard deviation. To compare the ACB and scintigraphy for DAT, relative distal proportion and gastric frequency, statistical analysis was performed using a Student's $t$-test and Spearman's rank correlation. Differences were considered significant at $P<0.001$.

Bland-Altman plots $^{27}$ were used to display the difference between the measurements by the two methods for each subject against their mean.

\section{RESULTS}

Figure 2 shows typical time-courses of IDF for scintigraphy and $\mathrm{ACB}$, as well as the corresponding sigmoidal curves. There was no significant difference in DAT obtained for scintigraphy and for ACB (Table 1). The relative proportion of the test meal in the distal region at DAT, obtained by means of ACB was significantly similar to the results obtained by scintigraphy (Table 1).

Fast Fourier transform revealed consistently clearly defined two dominant frequencies $(P>0.9)$ taken from the distal as well as the proximal stomach in all volunteers (Figs 3 and 4). Fast Fourier transform revealed consistently clearly defined two dominant frequencies $(P>0.9)$ taken from the distal as well as the proximal stomach (Figs 3 and 4) in all volunteers. 
A

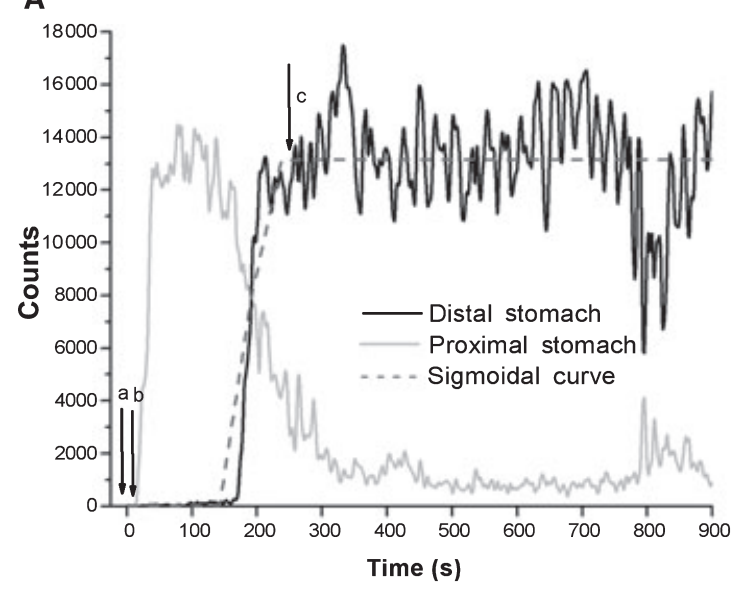

B

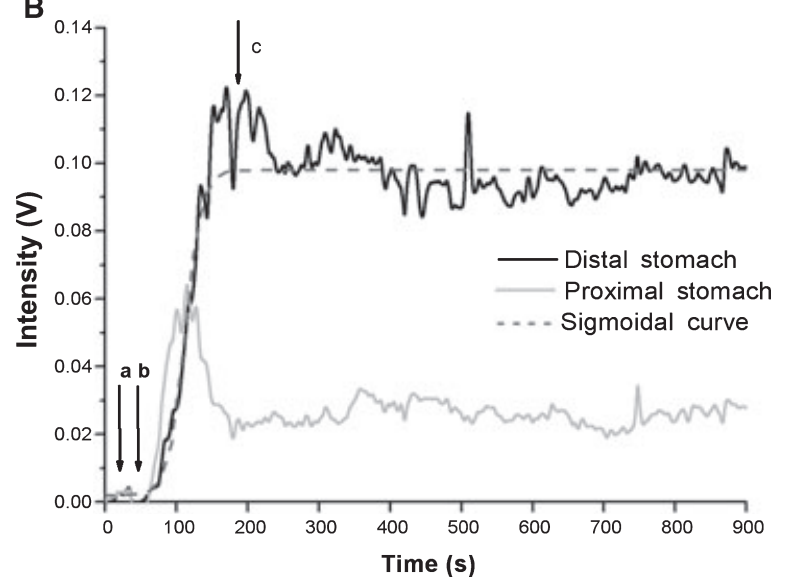

Figure 2 Examples of distribution of food (IDF) showing proximal and distal curves of distribution. (A) IDF profile recorded by scintigraphy and (B) IDF profile recorded by Alternating current Biosusceptometry. The arrows $\mathrm{a}$ and $\mathrm{b}$ indicate the beginning and the end of test meal ingestion respectively. Arrow c indicates when distal stomach signal reached $99 \%$ of the maximum value in the sigmoidal curves and determines distal accumulation time (DAT).

An interesting finding was a significative agreement between dominant frequencies around $1 \mathrm{cpm}$ recorded in proximal stomach and around $3 \mathrm{cpm}$ in distal stomach by scintigraphic and ACB (Table 1).
The Bland-Altman plots (Fig. 5) represent the gastric parameters evaluated by $\mathrm{ACB}$ and scintigraphy and showed that the pairs of measurements by the two methods had $95 \%$ limits of agreement. Calculating the correlation between both techniques (Table 1) was possible to note poor correlation in frequency parameter. However, this small correlation does not mean that two methods do not agree, ${ }^{27}$ since the difference $v s$ average of frequency values were within $95 \%$ limits of agreement.

\section{DISCUSSION}

Our study indicates that the assessment of intragastric distribution of a $60 \mathrm{~mL}$ liquid test meal by ACB yields results significantly similar to those obtained by scintigraphy. In fact, although the measurements with the two techniques have not been performed simultaneously, DAT values as well as IDF profiles were virtually identical.

In the present study, both scintigraphy and $\mathrm{ACB}$ showed that the fraction of the test meal retained in the proximal stomach at the end of the ingestion was smaller than reported in previous scintigraphy studies, ${ }^{9,11}$ and emptying of the proximal stomach was almost complete in a few minutes. This is not surprising since the volume of the test meal employed in our study is small in comparison to others, ${ }^{11,13,28,29}$ therefore conceivably demanding a lesser degree of proximal retention to prevent an excessive antral distension and/ or fast gastric emptying. It would be interesting to try to provide data on the relationship between meal size and IDF and accommodation by employing ACB technique.

Compelling evidence exists indicating that abnormal IDF is related to the genesis of dyspeptic symptoms, such as early satiety, fullness and nausea. ${ }^{9,14,29,30}$ As a consequence, gastroenterologists show increasing interest in simple, non-expensive, non-invasive and radiation-free methods of assessing IDF in clinical practice. Alternating current Biosusceptometry fulfils all these conditions and is as reliable as scintigraphy to perform this task.

Table 1 Gastric parameters evaluate by Alternating current Biosusceptometry (ACB) and scintigraphy

\begin{tabular}{|c|c|c|c|c|}
\hline & $\mathrm{ACB}$ & Scintigraphy & $\begin{array}{l}P \text {-value (ACB vs } \\
\text { scintigraphy) }\end{array}$ & $\begin{array}{l}\text { Correlation } \\
\text { coefficient }\end{array}$ \\
\hline Distal accumulation time (min) & $7.04 \pm 3.65$ & $6.93 \pm 3.25$ & $P<0.001$ & $r=0.72$ \\
\hline Relative distal proportion $(\%)$ & $80.03 \pm 7.19$ & $87.67 \pm 11.02$ & $P<0.001$ & $r=0.53$ \\
\hline Frequency in proximal stomach $(\mathrm{cpm})$ & $0.98 \pm 0.06$ & $1.01 \pm 0.01$ & $P<0.001$ & $r=0.76$ \\
\hline Frequency in distal stomach $(\mathrm{cpm})$ & $3.06 \pm 0.08$ & $3.07 \pm 0.11$ & $P<0.001$ & $r=0.37$ \\
\hline
\end{tabular}

Data were expressed as mean $\pm \mathrm{SD}$. 
A
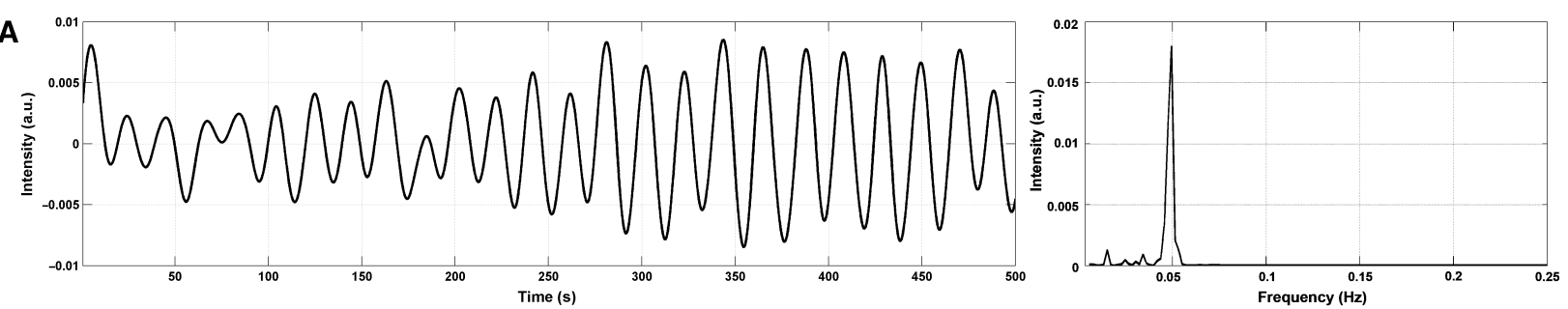

B
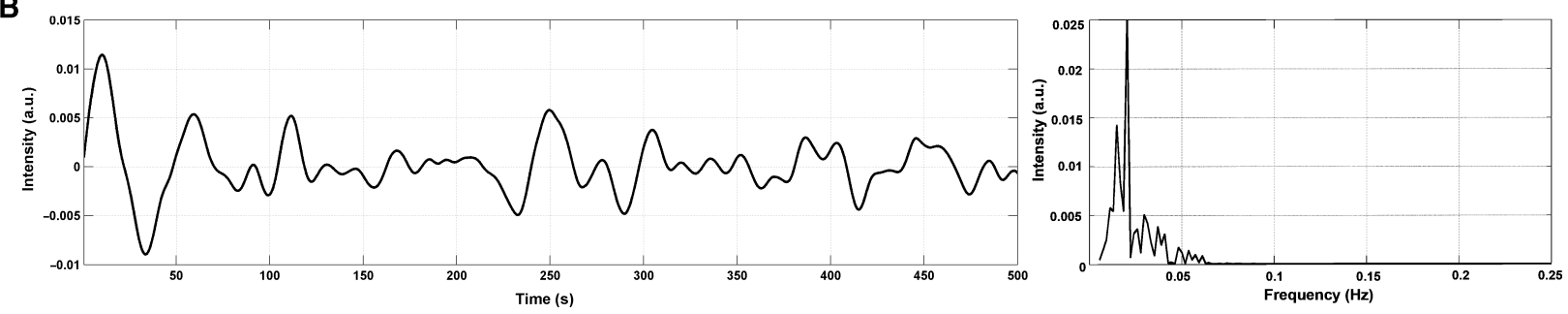

Figure 3 Spectral analysis of biomagnetic tracings for two gastric regions. (A) Distal stomach tracing shows an intense dominant frequency of $0.0500 \mathrm{~Hz}(3 \mathrm{cpm})$ which was verified in the respective fast Fourier transform (FFT). (B) In proximal region, it was possible to verify predominantly a frequency pattern of $0.0165 \mathrm{~Hz}(1 \mathrm{cpm})$ in the registered signal and its FFT.

A

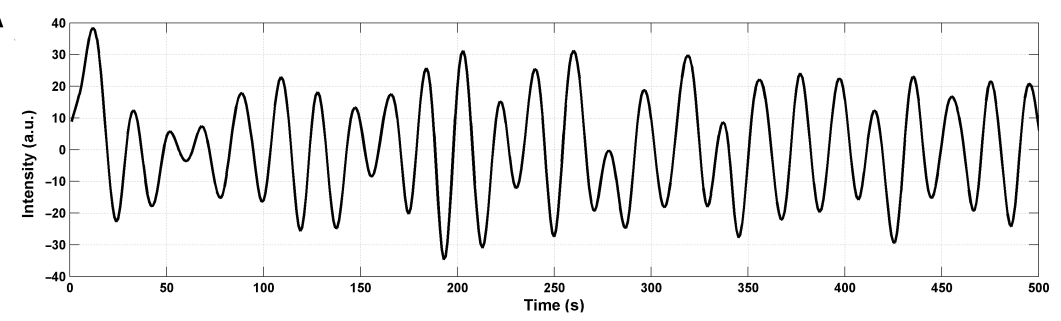

B

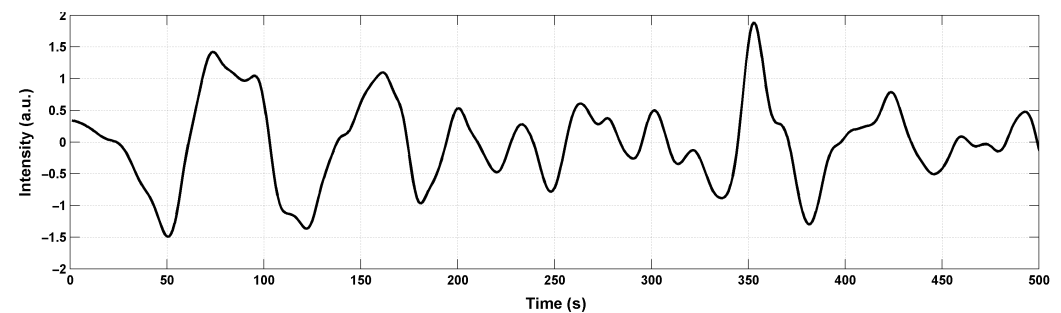

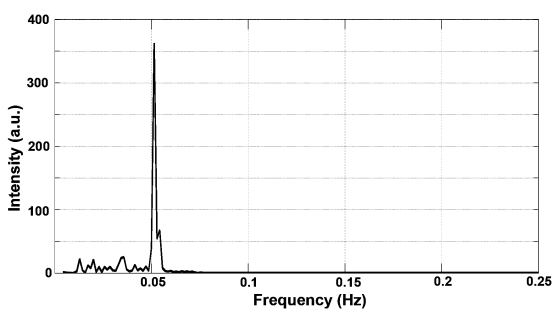

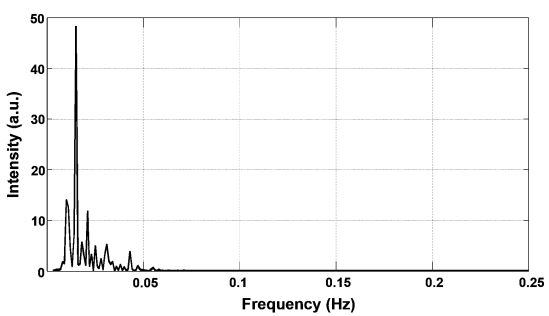

Figure 4 Spectral analysis of scintigraphic recordings for two gastric regions. (A) In distal stomach are demonstrate an intense dominant frequency of $0.0500 \mathrm{~Hz}(3 \mathrm{cpm})$ which was verified in fast Fourier transform (FFT). (B) In proximal region was recorded a frequency pattern of $0.0165 \mathrm{~Hz}(1 \mathrm{cpm})$ that was clearly identified in the FFT.

The positioning of the magnetic sensors (abdominal surface) and the ROIs (images) was based on the two regions clearly distinguished during the meal intragastric displacement; thus, there was decrease of distribution curve in the proximal stomach while concomitantly there was increase in the distal. The distribution of the test meal in each region expressed as percentage allows a more objective quantification of the IDF and emphasizes the similar profile between both techniques. It is noteworthy that respiratory artefacts were conspicuous in all scintigraphic and magnetic recordings, and were removed by digital filter.

Barostat still remains the gold standard for proximal stomach accommodation studies in humans, but the utility of this technique in the clinical setting is limited due to its invasiveness. ${ }^{8}$ The scintigraphy is a suitable technique for IDF studies, nevertheless involves exposure to ionizing radiation. ${ }^{6,15}$ Since its original development, $\mathrm{ACB}$ has been optimized in 
A

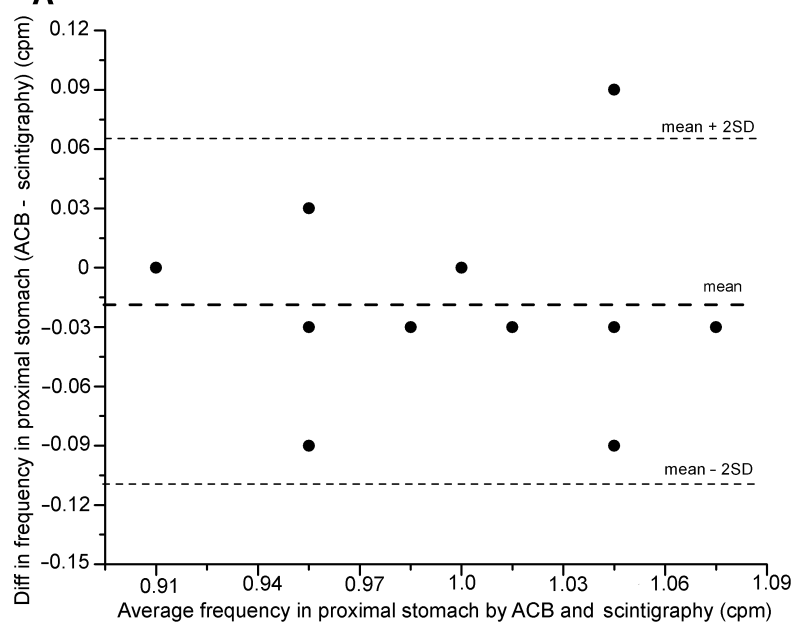

B

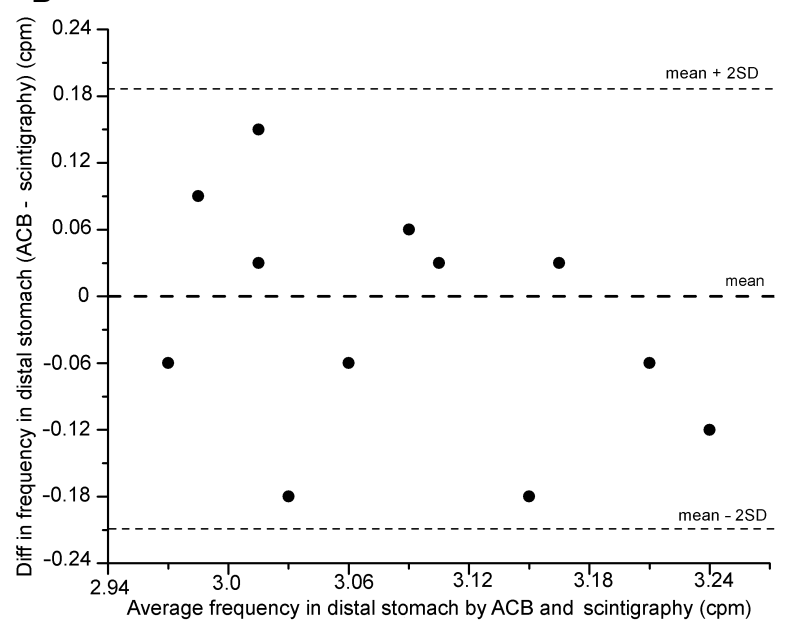

C

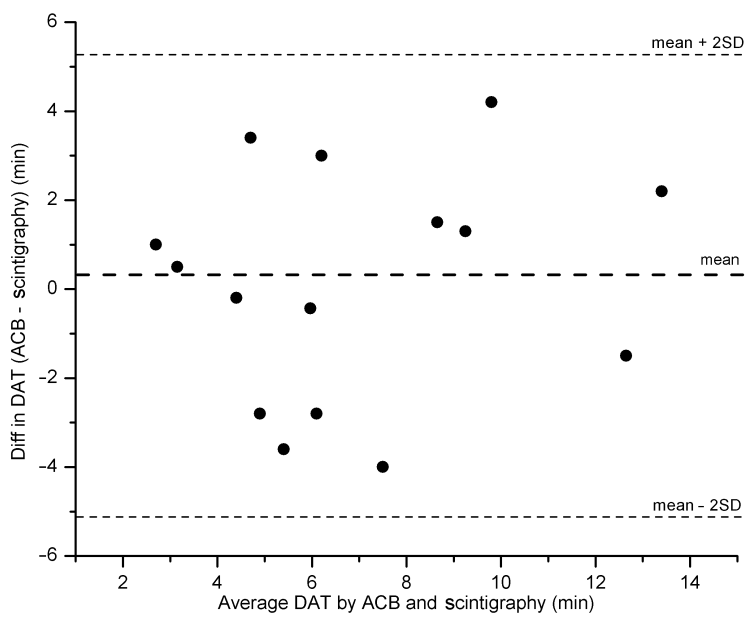

D

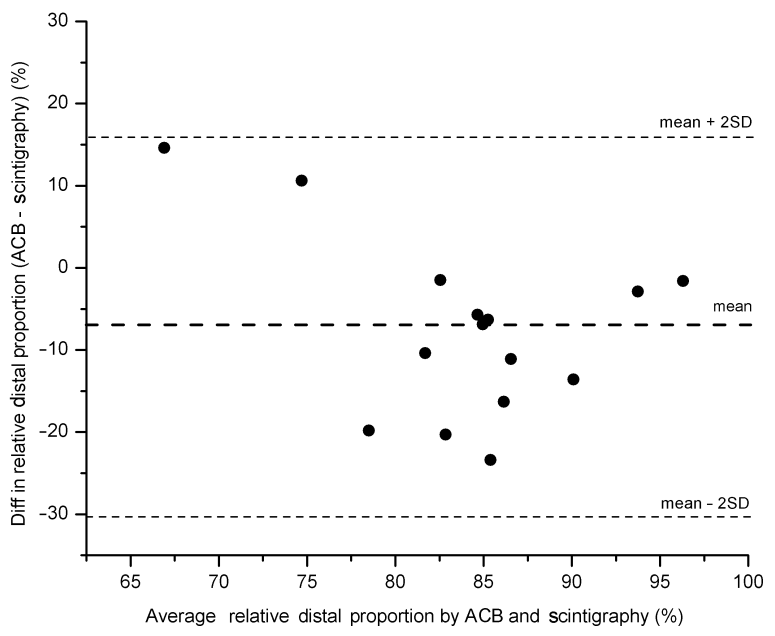

Figure 5 Bland-Altman plots of all gastric parameters evaluated by Alternating current Biosusceptometry and scintigraphy. (A) and (B) illustrate the two frequencies recorded, while (C) shows the distal accumulation time and (D) the relative distal proportion.

instrumental issues to meet the needs required in GI motility studies. Ferrite concentration in the meal test was well above that concentration necessary to yield a high signal/noise ratio, even in obese subjects. Multisensor ACB technique was able to characterize with accuracy IDF process with low calorie and small volume meal and to recorder gastric motility in real time.

An important finding of this study was that postprandial phasic motor activities were recorded by both $\mathrm{ACB}$ and scintigraphy. Discharge from the gastric pacemaker usually occurs at a frequency of $0.05 \mathrm{~Hz}$ and was easily recognized by visual inspection of the recordings of both scintigraphy and $\mathrm{ACB}$, and clearly demonstrated by the corresponding spectral analysis. These results are very similar to the previous studies on gastric antral motility using scintigraphy. ${ }^{31,32}$
Interestingly, when the scintigraphic and susceptometric procedures of signals analysis from the distal stomach were applied to more proximal points in the epigastrium, which corresponds to the proximal stomach, a different phasic activity was consistently recorded for both techniques and for all volunteers. The spectral analysis revealed a dominant frequency of about $0.016 \mathrm{~Hz}(1 \mathrm{cpm})$, virtually identical to the frequency of the so-called 'volume waves' recorded in studies employing barostat technique., ${ }^{3,22}$

The motor activity around $1 \mathrm{cpm}$ on proximal stomach in dogs and humans was reported by different techniques. ${ }^{2,5,23,33,34}$ However, this frequency has not been extensively documented due to mainly methodological issues, such as differences in barostat systems $^{3}$ and/or filter parameters employed in data analysis. 
Recently, evidence was produced that unsuppressed postprandial phasic activity in the proximal stomach plays a pathophysiological role in functional dyspepsia. ${ }^{23}$ This finding increases the demand for reliable, easy to perform and comfortable techniques to accelerate the acquisition of knowledge on proximal stomach motility in the clinical setting, ${ }^{35}$ and our data suggest that both scintigraphy and $\mathrm{ACB}$ are promising techniques for this purpose.

\section{ACKNOWLEDGMENTS}

The authors would like to thank the Brazilian agencies: FAPESP/PRONEX and CAPES for financial support.

\section{REFERENCES}

1 Rao SSC, Vemuri S, Harris B, Schulze K. Fundic ballon distension stimulates antral and duodenal motility in man. Dig Dis Sci 2002; 47: 1015-9.

2 Quigley EMM. Gastric and small intestinal motility in health and disease. Gastroenterol Clin North Am 1996; 25: 113-45.

3 Ahluwalia NK, Thompson DG, Barlow J. Effect of distension and feeding on phasic changes in human proximal gastric tone. Gut 1996; 39: 757-61.

4 Schepper HU, Cremonini F, Chitkara D, Camilleri M. Assessment of gastric accommodation: overview and evaluation of current methods. Neurogastroenterol Motil 2004; 16: 275-85.

5 Zwart IM, Mearadji B, Lamb HJ et al. Gastric motility: comparison of assessment with real-time MR imaging or barostat measurement - initial experience. Radiology 2002; 224: 592-7.

6 Schwizer W, Steingötter A, Fox M et al. Non-invasive measurement of gastric accommodation in humans. Gut 2002; 51: 59-62.

7 Kuiken SD, Samsom M, Camilleri M et al. Development of a test to measure gastric accommodation in humans. Am I Physiol 1999; 277: G1217-21.

8 Ropert A, Varannes SB, Bizais Y, Rozé C, Galmiche JP. Simultaneous assessment of liquid emptying and proximal gastric tone in humans. Gastroenterology 1993; 3105: 66774.

9 Troncon LEA, Bennett RJ, Ahluwalia NK, Thompson DG. Abnormal intragastric distribution of food during gastric emptying in functional dyspepsia patients. Gut 1994; 35: 327-32.

10 Feinle-Bisset C, Vozzo R, Horowitz M, Talley NJ. Diet, food intake, and disturbed physiology in the pathogenesis of symptoms in functional dyspepsia. Am I Gastroenterol 2003; 99: 170-81.

11 Troncon LEA, Rosa-E-Silva L, Oliveira RB, Iazigi N, Gallo L Jr, Foss MC. Abnormal intragastric distribution of a liquid nutrient meal in patients with diabetes mellitus. Dig Dis Sci 1998; 43: 1421-9.

12 Troncon LEA, Thompson DG, Ahluwalia NK, Barlow J, Heggie L. Relations between upper abdominal symptoms and gastric distension abnormalities in dysmotility like functional dyspepsia and after vagotomy. Gut 1995; 37: 17-22.

13 Tack J, Piessevaux H, Coulie B, Caenepeel P, Janssens J. Role of impaired gastric accommodation to a meal in functional dyspepsia. Gastroenterology 1998; 115: 134652.

14 Piessevaux H, Tack J, Walrand S, Pauwels S, Geubel A. Intragastric distribution of a standardized meal in health and functional dyspepsia: correlation with specific symptoms. Neurogastroenterol Motil 2003; 15: 447-55.

15 Feinle C, Kunz P, Boesiger P, Fried M, Schwizer W. Scintigraphic validation of a magnetic resonance imaging method to study gastric emptying of a solid meal in human. Gut 1999; 44: 106-11.

16 Corá LA, Romeiro FG, Stelzer M et al. Biosusceptometry in the study of drug delivery. Adv Drug Deliv Rev 2005; 57: 1223-41.

17 Corá LA, Romeiro FG, Paixão FC et al. Enteric coated magnetic HPMC capsules evaluated in human gastrointestinal tract by AC Biosusceptometry. Pharm Res 2006; 23: 1809-16.

18 Miranda JRA, Oliveira RB, Souza PL, Braga FJH, Baffa O. A novel biomagnetic method to study gastric antral contractions. Phys Med Biol 1997; 42: 1791-9.

19 Miranda JRA, Baffa O, Oliveira RB, Matsuda NM. An AC biosusceptometer to study gastric emptying. Med Phys 1992; 19: 445-8.

20 Oliveira RB, Baffa O, Troncon LEA, Miranda JRA, Cambrea $C R$. Evaluation of a biomagnetic technique for measurement of orocaecal transit time. Eur I Gastroenterol Hepatol 1996; 8: 491-5.

21 Moraes R, Corá LA, Américo MF, Oliveira RB, Baffa O, Miranda JRA. Measurement of gastric contraction activity in dogs by means of AC Biosusceptometry. Physiol Meas 2003; 24: 337-45.

22 Allocca M, Penagini R. Effect of phasic contractions and tone of the proximal stomach on triggering of transient lower esophageal sphincter relaxation. Dig Dis Sci 2004; 49: 710-4.

23 Simrén M, Vos R, Janssens J, Tack J. Unsupressed postprandial phasic contractility in the proximal stomach in functional dyspepsia: relevance to symptoms. Am J Gastroenterol 2003; 98: 2169-75.

24 Romeiro FGR, Corá LA, Andreis U et al. A novel biomagnetic approach to study caecocolonic motility in humans. Neurogastroenterol Motil 2006; 18: 1078-83.

25 Frei EH, Gunders E, Pajewsky M, Alkan WJ, Eshcher J. Ferrites as contrast material for medical X-ray diagnosis. J Appl Phys 1968; 39: 99-101.

26 Hadi NA, Giouvanoudi A, Morton R, Horton PW, Spyrou NM. Variations in gastric emptying times of three stomach regions for simple and complex meals using scintigraphy. IEEE Trans Nucl Sci 2002; 49: 2328-31.

27 Bland JM, Altman DG. Statistical methods for assessing agreement between two methods of clinical measurement. Lancet 1986; 8: 307-10.

28 Collins PJ, Horowitz M, Chatterton BE. Proximal, distal and total stomach emptying of a digestible solid meal in normal subjects. Br J Radiol 1988; 61: 12-8.

29 Boeckxstaens GE, Hirsch DP, Kuiken SD, Heisterkamp $\mathrm{SH}$, Tytgat GNJ. The proximal stomach and postprandial 
symptoms in functional dyspeptics. Am I Gastroenterol 2002; 97: 40-8.

30 Tack I, Bisschops R, Sarnelli G. Pathophysiology and treatment of functional dyspepsia. Gastroenterology 2004; 127: 1239-55.

31 Urbain JL, Vekemans MC, Parkman H et al. Dynamic antral scintigraphy to characterize gastric antral motility in functional dyspepsia. J Nucl Med 1995; 36: 1579-86.

32 Troncon LEA, Herculano JR, Savoldelli RD, Moraes ER, Secaf M, Oliveira RB. Relationship between intragastric food maldistribution disturbances of antral contractility, and symptoms in functional dyspepsia. Dig Dis Sci 2006; 51: $517-26$.
33 Lind LF, Douthie HL, Schlegel JF, Code CF. Motility of the gastric fundus. Am I Physiol 1961; 201: 197-202.

34 Koch KL, Stern RM. Nausea and vomiting and gastric dysrhythmias. In: Chen JZ, McCallum RW, eds. Electrogastrography: Principles and Applications. Electrogastrography: Principles and Applications. New York: Raven Press Ltd, 1994: 309-30.

35 Lee KJ, Vos R, Janssens J, Tack J. Differential effects of baclofen on lower oesophageal sphincter pressure and proximal gastric motility in humans. Aliment Pharmacol Ther 2003; 18: 199-207. 